\title{
acción expansiva de la ilita en Vélez-Málaga
}

\author{
FERNANDO OLIVEROS RIVES, Ingeniero de Caminos \\ Y AUGUSTO VIZCAINO MARTINEZ, Ingeniero Aeronáutico
}

\section{$478-4$}

\section{simopeis}

En la zona costera central de la provincia de Málaga son muy frecuentes los casos de existencia de suelos expansivos y se han escogido los situados al S. E. de Vélez-Málaga, para realizar sobre ellos un estudio detallado en el presente trabajo. Sobre tales suelos están edificados unos bloques de viviendas que se encuentran gravemente dañados, como consecuencia de la acción de dichos suelos sobre su cimentací́n, daños que se ofrecen a la vista del observador.

La constitución arcillosa de tales suelos es debida a la formación geológica del pizarral silúrico que la bordea. El suelo consiste en unas arcillas azules que adopt

De los análisis roentgenográficos efectuados se deduce que estas arcillas están constituidas fundamentalmente por ilita, carbonato cálcico y cuarzo en la proporción de $1: 2: 3$, además de montmorillonita, dolomita y otros elementos. Así, pues, la ilita es el mineral arcilloso predominante, hecho que ha sido confirmado por el ańlisis térmico diferencial y, por tanto, es el elemento que ha provo. los efectos expansivos del suelo.

La potencia de hinchamiento se observa más elevada en los estratos superiores, correspondientes a las arcillas amarillas, que en las azules de base. Este hecho es, en parte, debido a la riqueza de carboneto cáleico que posee la arcilla azul, y que llega a ser superior al doble de la superficial. La abundancia de $\mathrm{CO}_{5} \mathrm{Ca}$ en la arcilla azul reduce su indice de plasticidad y, por tanto, disminuye la actividad expansiva del suelo.

El valor máximo deducido para el potencial de hinchamiento en el Laboratorio, ha sido del $9 \%$, y en el resto, valores sensibles. Estos valores originaron en la cimentación de las edificaciones correspondientes, importantes esfuerzos verticales. Las presiones ejercidas en el caso más desfavorable, aleanzaba valores de $0,750 \mathrm{~kg} / \mathrm{cm}^{2}$. Este fenómeno ha sido el productor de los daños observados.

Como solución de cimentación se recomienda cimentar con hormigón armado sobre la marga azul, apoyando sobre gravas apisona. das, que absorberían la expansión volumétrica del suelo, neutralizando así tales efectos si se produjesen. La cimentación ha de (a) que podrían provocar la iniciación de su proceso de hinchamiento.

\section{Antecedentes}

En la faja costera central de la provincia de Málaga hemos encontrado con frecuencia edificios fisurados por la acción de los suelos expansivos, así como la existencia de éstos en numerosos reconocimientos. Se han escogido los situados al SE. de Vélez-Málaga, para realizar sobre ellos un estudio detallado.

Sobre dichos suelos hay unos bloques de viviendas, en los que se pueden observar numerosas grietas, aun cuando son de reciente construcción. Ello hizo pensar que podría ser defecto de la cimentación, y como, por otra parte, se proyectaba construir en las zonas adyacentes otros grupos de casas, es por lo que se decidió efectuar, en el mes de abril de 1962, un amplio reconocimiento de esta zona por medio de sondeos y extracción de muestras inalteradas, con objeto de analizar, detenidamente, las características del suelo $\mathrm{y}$, a la vista de los resultados, considerar la forma de reparar los edificios ya construidos y decidir sobre el tipo de cimentación que se debería adoptar para las nuevas construcciones (fig. 1).

\section{Desceripción \\ clel femómema}

Las construcciones de que tratamos, ejecutadas en los años 1957 1960, poseían una o dos plantas y la cimentación consistía en un macizo de hormigón ciclópeo en base, de 60 centímetros de ancho por $30 \mathrm{~cm}$ de alto, en donde se apoyaba una
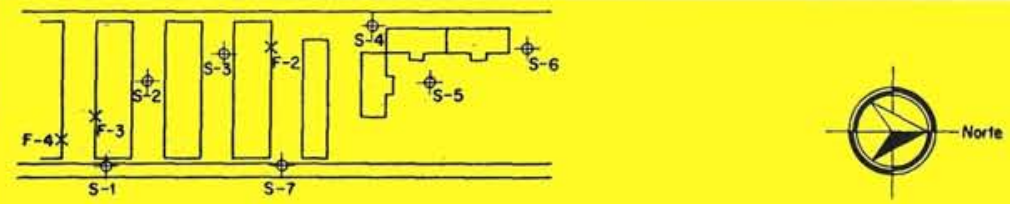

$S-K=$ SONDEO
$F-K=$ FOTOGRAFIA

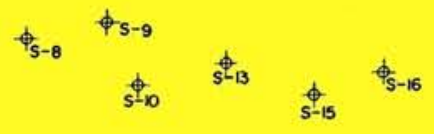

s-11 $\underset{s-12}{\Phi}$ s-18 $\$_{s-19}$ s-17

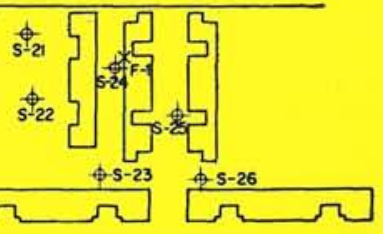

Fig. 1

(Escala, $1: 2.000$ ) 
viga de la misma anchura y $30 \mathrm{~cm}$ de canto, armada con $2 \varnothing 14$ por cada cara; sobre esta viga iba otra, también de hormigón en masa de $40 \mathrm{~cm}$ de canto. El hormigón superior e inferior a la viga armada descrita era pobre, de unos $175 \mathrm{~kg} / \mathrm{m}^{3}$. Los materiales empleados en los alzados eran de calidad corriente y las fábricas, perfectamente aceptables no sólo en su apariencia, sino en su estado resistente.

La expansión se produce en sentido tridimensional. Por lo que se refiere a las tensiones horizontales, cuando no hay cimentación, quedan contrarrestadas entre sí, y cuando existe, provocan una coacción sobre el cimiento. En cuanto a la tensión vertical se manifiesta de dos formas: El empuje de base actuando sobre la superficie en planta de la cimentación, y el de fricción en las caras laterales de la misma; naturalmente, que esta fricción no puede considerarse como de fábrica con suelo, pues la adhesión entre ambos permite contar más bien con el valor de la cohesión de éste. Los dos efectos son verticales y sumados producen los movimientos del edificio.

Estas edificaciones han sufrido unos esfuerzos verticales producidos por el suelo, que las ha sometido a modo de viga sujeta a cargas repartidas, y al deformarse, en la apertura encuentra cohibido su movimiento por el empuje pasivo, ejercido por las zonas de suelo en contacto con la misma; este efecto produce en dichas zonas un cierto grado de inmovilidad.

Los bloques se encuentran gravemente afectados por la formación de grietas, que podemos clasificarlas en dos tipos: el sistema primario compuesto por fisuras verticales (figs. 2 y 3 ) que se inician, en general, en la línea neutra y ascienden en sentido vertical hasta la coronación, abriéndose tanto más con la cota (la acción climática todavía favorecerá más este efecto), y el sistema derivado que depende, además, de la influencia del empotramiento en el suelo de la estructura espacial que constituye la edificación, la cual ofrece unos pórticos a modo de formas elementales resistentes, en ambos sentidos. Los edificios no estaban calculados para resistir la deformación impuesta por las presiones ejercidas por el suelo, que han originado las manifestaciones de rotura indicadas, como consecuencia de un reparto tensional incompatible con la correspondiente sección resistente. El efecto, como puede comprenderse, es mayor en las partes centrales de la edificación que en los extremos, y si en algunos casos la estructura pudo resistir la tensión, las roturas afectan entonces a las partes marginales (fig. 4) o a los elementos débiles superficiales, como las aceras (fig. 5). La grieta de esta acera se habría alejado paralelamente hacia la calzada si la misma hubiese tenido pavimento, pero durante el desarrollo del fenómeno permaneció sin él.

Como se sabe, cuanto menor es la altura del edificio, más sensible es el fenómeno, puesto que los empujes verticales no quedan compensados con el peso propio $\mathrm{y}$, por tanto, mayores son los daños que sufre.

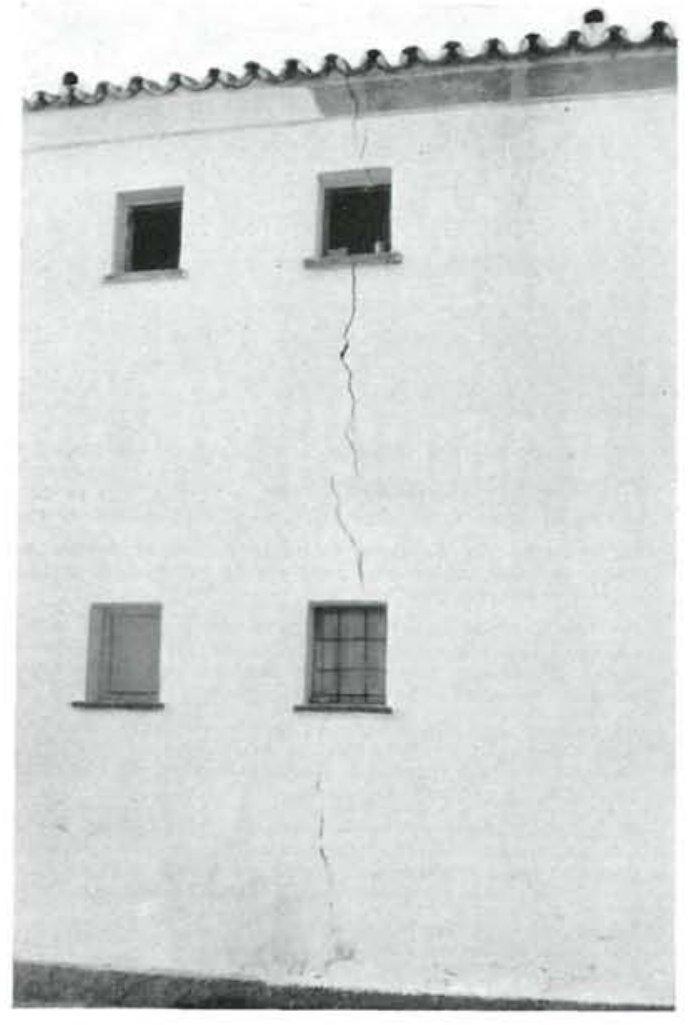

Fig. 2

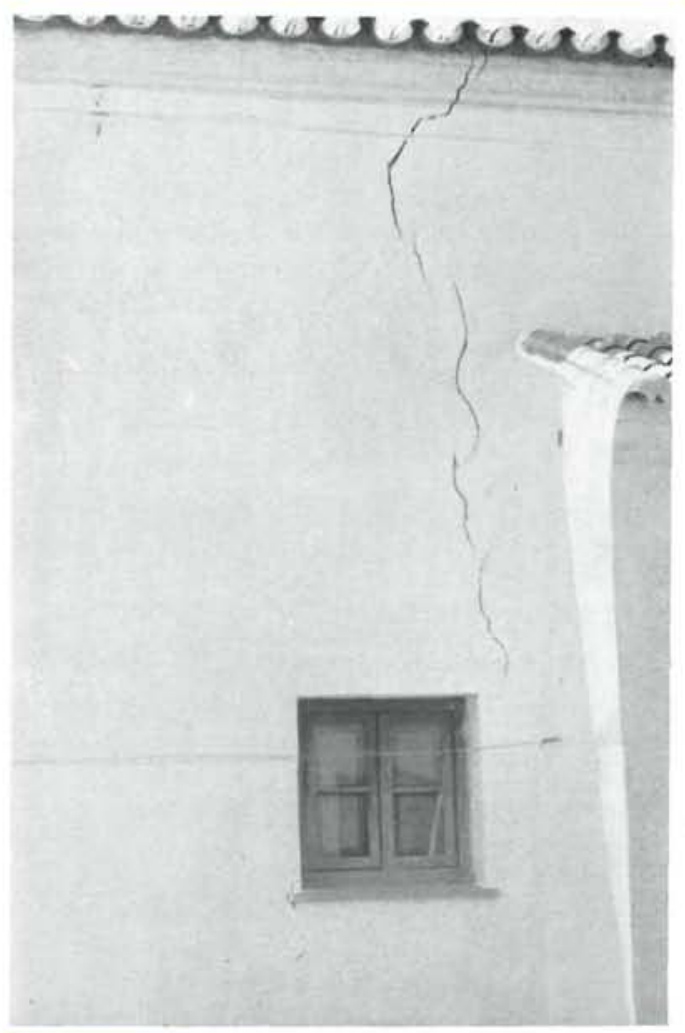

Fig. 3 


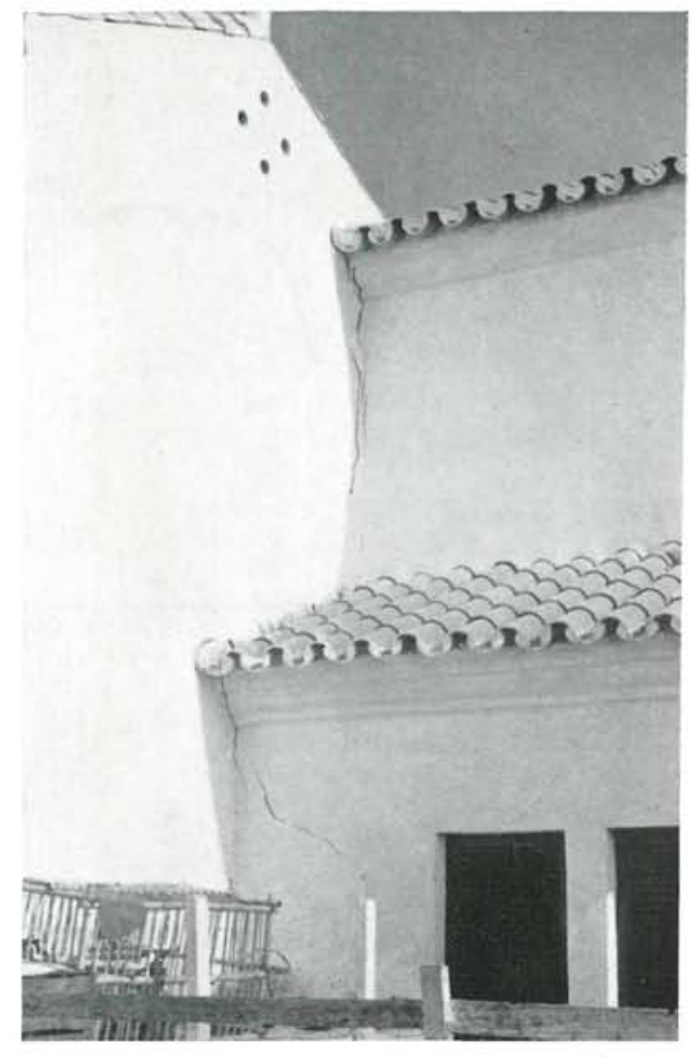

Fig. 4

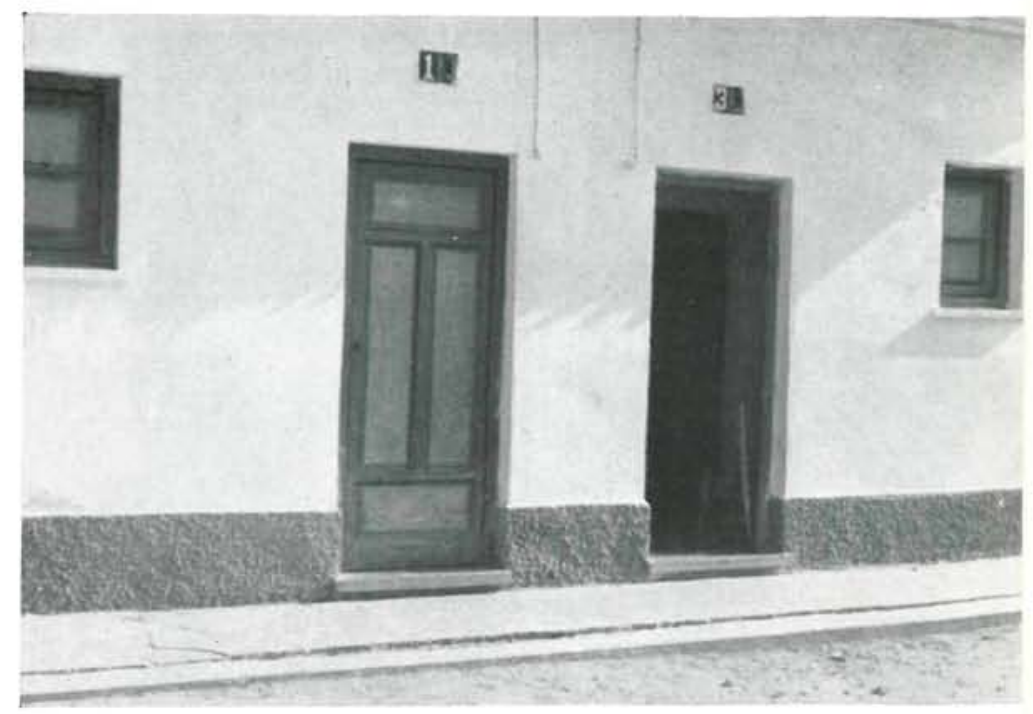

Fig. 5

\section{Aspecto geológico}

Los terrenos de formación de esta zona están constituidos por arcillas diluviales del cuaternario; y el hecho de que esta zona se encuentra bordeada por pizarras silúricas obliga a pensar que dichas arcillas se han formado a expensas de aquéllas por sedimentación de materiales procedentes de la descomposición y transformación de las mismas.

Tales arcillas están recubiertas en algunos sectores por un lecho superficial, formado por acarreos que han actuado de manto de cobertura, preservándolas en cierto modo, ya que, si bien la parte superficial del manto está oxidada y posee un color amarillento, la presencia del citado lecho ha reducido la acción oxidante en profundidad. El color inicial de las arcillas es azulado, y entre ambos mantos existe una zona de transición constituida por arcilla gris, correspondiente al primer período de alteración, que, una vez completado, manifestará el color amarillo de las arcillas superficiales. Un perfil característico puede observarse en la figura 6 .

\section{Aspecto geotécinico del suelo}

Con objeto de obtener información que permitiera resolver el problema, se ha llevado a cabo una intensa exploración de las áreas edificadas y por edificar, efectuada mediante sondeos, cuya distribución en planta se indica en la figura 1. Partiendo de estos sondeos de reconocimiento se han obtenido muestras inalteradas que han permitido la identificación y clasificación de los suelos que forman los estratos, así como el estudio de sus características geomecánicas. Desde el punto de vista geotécnico están formados por dos estratos: el más superficial, no continuo, puesto que sólo aparece en las áreas no edificadas, está constituido por gravas más o menos arenosas, de espesor máximo 1,5 m; el segundo, y más interesante, está formado por varios tipos de arcillas que tienen todas el mismo origen, si bien se registran alteraciones producidas por agentes atmosféricos y oxidaciones que afectan no sólo al color, sino al comportamiento físico-químico.

\section{Composición química de las arcillas}

Se ha seguido el método de Jacob para los silicatos, y se seleccionaron tres muestras representativas que dieron el siguiente resultado: 


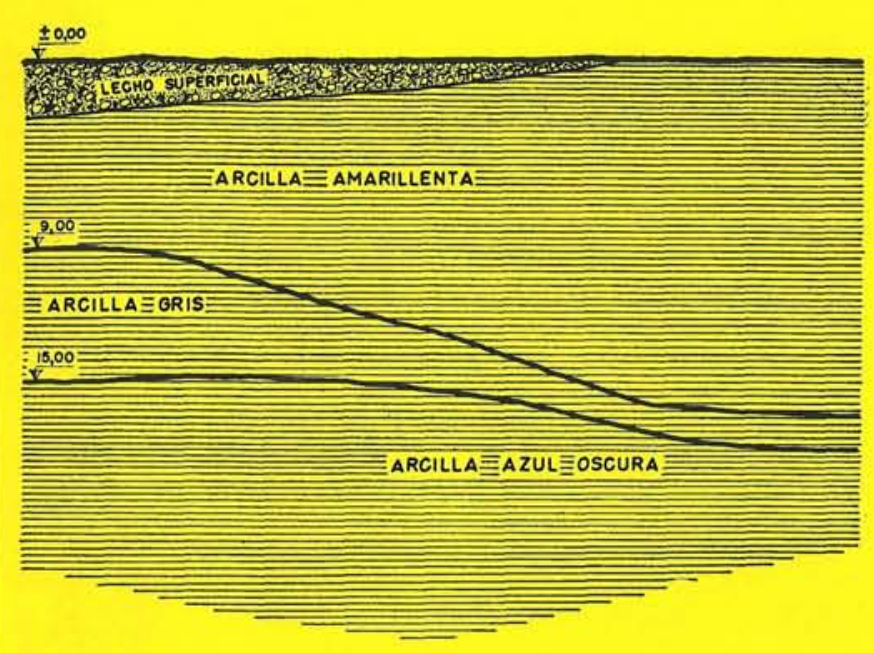

Fig. 6

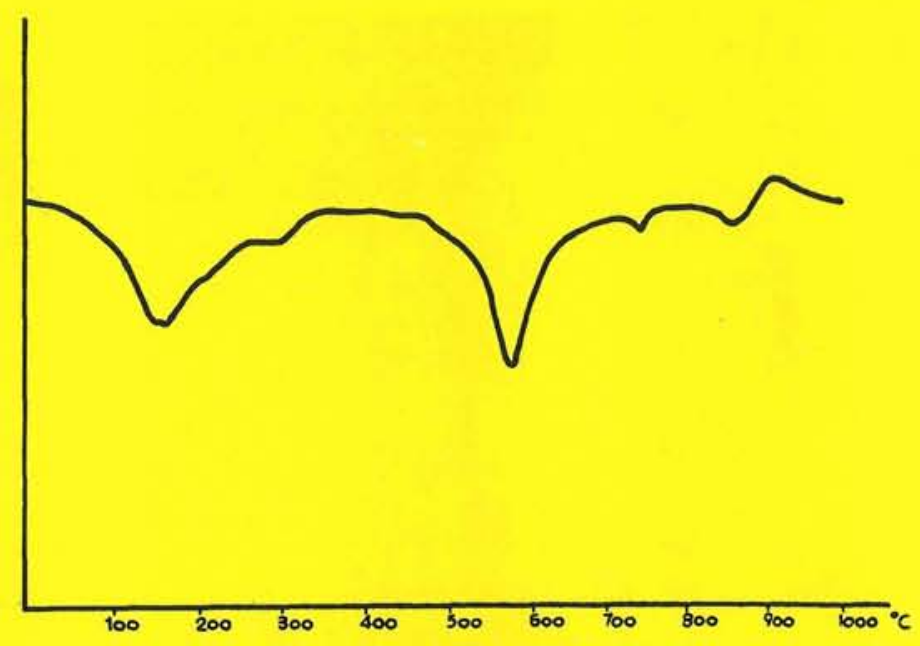

Fig. 7

\begin{tabular}{|c|c|c|c|}
\hline oxidos \% & $\begin{array}{l}\text { Sondeo } 10 \\
\text { Muestra } 1\end{array}$ & $\begin{array}{l}\text { Sondeo } 10 \\
\text { Muestra } 3\end{array}$ & $\begin{array}{c}\text { Sondeo } 1 \\
\text { Muestra } 1\end{array}$ \\
\hline 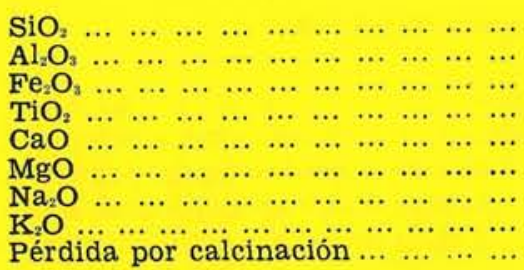 & $\begin{array}{r}50,7644 \\
16,6883 \\
4,2470 \\
0,8893 \\
9,3734 \\
2,9779 \\
2,0988 \\
0,8094 \\
12,1515\end{array}$ & $\begin{array}{r}49,8369 \\
16,3651 \\
4,9244 \\
1,0086 \\
8,5632 \\
1,9875 \\
2,9269 \\
0,9394 \\
13,4480 \\
\end{array}$ & $\begin{array}{r}52,3297 \\
12,7706 \\
3,9907 \\
0,8780 \\
11,7530 \\
1,6263 \\
2,1451 \\
0,7383 \\
13,7683 \\
\end{array}$ \\
\hline & 100 & 100 & 100 \\
\hline
\end{tabular}

Era interesante conocer la variación del contenido de OCa con la profundidad, y para ello se hizo la determinación con tres muestras de un mismo sondeo, obteniéndose los siguientes resultados:

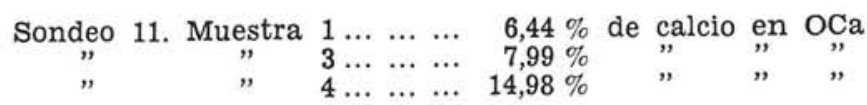

Así, pues, las zonas superficiales poseen menos OCa que las inferiores, donde el contenido llega a ser más del doble.

En el Departamento de Silicatos del Patronato Juan de la Cierva se ha hecho un análisis mediante rayos $\mathrm{X}$, resultando que casi el $50 \%$ del $\mathrm{SiO}_{2}$, se encuentra en forma de sílice libre, probablemente en la fracción mayor de 2. Deducido este valor de la sílice, la relación $\mathrm{SiO}_{2} / \mathrm{Al}_{2} \mathrm{O}_{3}$ toma valores que pueden corresponder tanto a minerales caoliníticos como ilíticos y montmorilloníticos.

El análisis roentgenográfico fue efectuado por dicho laboratorio en un difractómetro Philips, con radiación $\mathrm{K}_{x}$ de cobre y registro gráfico, encontrándose ilita, carbonato cálcico y cuarzo en la proporción $1: 2: 3$, aparte de montmorillonita, caolinita y dolomita en menores proporciones.

El análisis térmico diferencial se realizó en hornos eléctricos con pares de cromel-alumel, observándose, predominantemente, los efectos térmicos debidos a la ilita, que puede considerarse como el mineral arcilloso más predominante. La figura 7 muestra una de las curvas obtenidas, que puede considerarse como tipo.

Como se sabe, a igualdad de peso, la montmorillonita es el mineral de mayor potencia expansiva, pero cantidades superiores de ilita pueden provocar efectos expansivos análogos. 


\section{Características de identificación y clasificación}

$\mathrm{El}$ aspecto que presentaban las muestras al ser extraídas de sus envolturas, era el de suelos de consistencia natural blanda, muy próxima al límite plástico. En la figura 8 quedan reflejados en profundidades los valores deducidos para las muestras de un sondeo que, a efectos generales, pueden considerarse como característicos.

Las propiedades de identificación y clasificación de las diversas muestras remitidas al Laboratorio, se indican en el cuadro I; los ensayos realizados que figuran se han efectuado siguiendo las normas ASTM. En la figura 9 se representa la distribución del tamaño de las partículas de tres muestras correspondientes al sondeo número 11 ; todas las demás muestras ensayadas tienen una granulometría análoga, por lo que pueden considerarse como representativas del área explorada. En este gráfico se comprueba que el $100 \%$ de las partículas pasa por el tamiz número 200 ASTM, y que, aproximadamente, el $50 \%$ son partículas con diámetro equivalente inferior a 0,005 milímetros.

Los resultados del límite líquido y del índice de plasticidad de todas las muestras estudiadas, se han representado en la carta de plasticidad de Casagrande (fig. 10), y los puntos que los representan están alineados según una recta con igual pendiente que la línea $\mathrm{A}$, poniendo de manifiesto la identidad de origen ya señalada. En esta representación el índice de plasticidad varía entre 20 y $40 \%$, y el límite líquido queda entre 40 y $60 \%$.

El índice de fluidez tiene un valor medio muy próximo a 1 , lo que demuestra que la con-

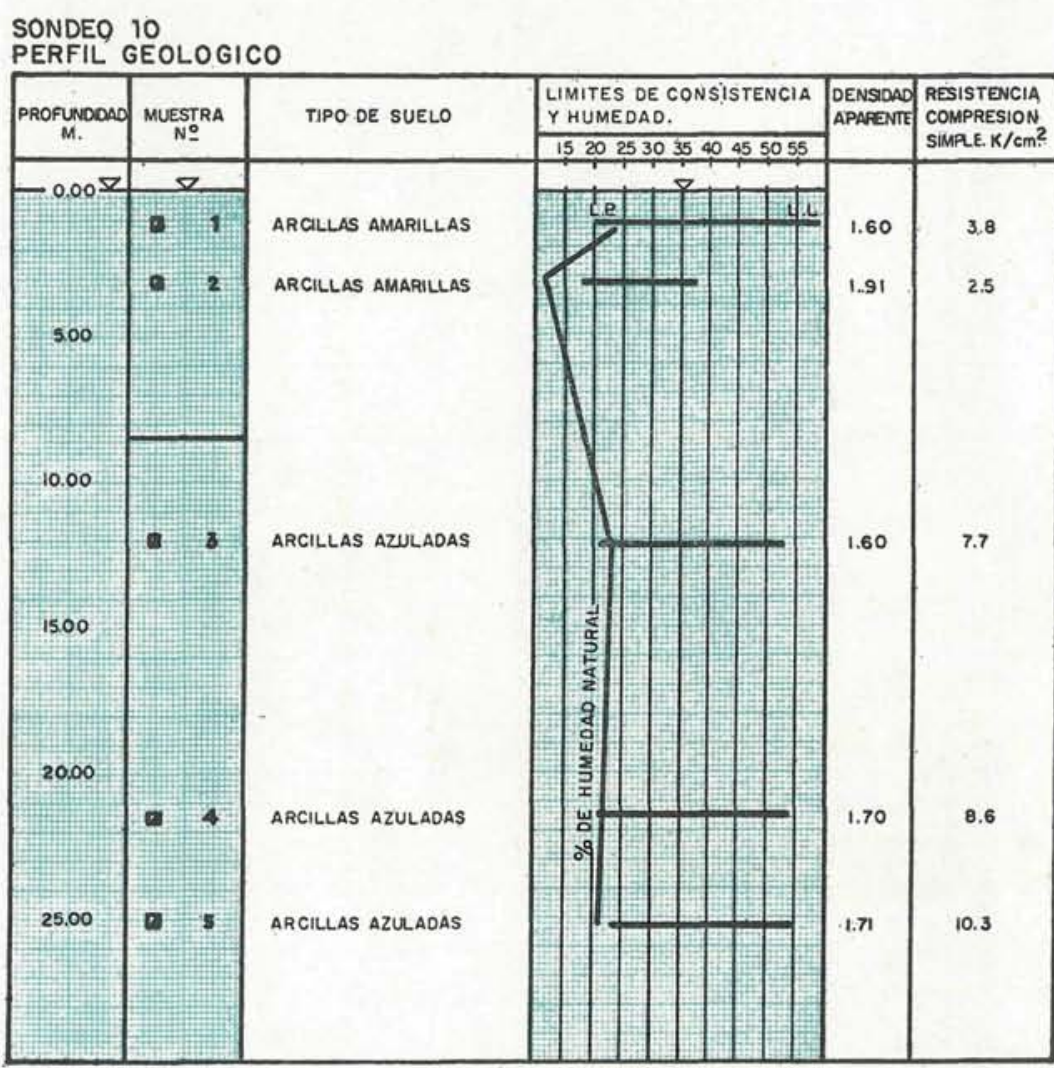

Fig. 8 sistencia es plástica dura, que concuerda con su estado natural, del cual ya hemos hablado en los párrafos anteriores.

Como consecuencia de estas propiedades físicas y, de acuerdo con la clasificación de Casagrande, podemos decir que estos suelos son del tipo CI y están constituidos por arcillas carentes de materia orgánica, con plasticidad media, ya que los puntos se encuentran en torno del límite líquido 50.

El límite de contracción de las muestras ensayadas de diversos sondeos varía entre 14 y 16 por ciento.

\section{Densidad y humedad}

Las densidades aparentes de las muestras son relativamente débiles, pero con una marcada tendencia densificante en profundidad (columna 9 del cuadro 1 ).

El contenido de agua natural de estas arcillas en cotas inferiores a los $5 \mathrm{~m}$ está comprendido, prácticamente, entre 20 y $25 \%$, si bien hay que hacer alguna salvedad en las áreas edificadas, en las que alcanza valores inferiores a estos límites. En las áreas no edificadas la humedad es mucho más homogénea en profundidad y extensión, posiblemente debido a la protección producida por el lecho superficial de acarreos. El grado de saturación llega en las más profundas hasta el 95 por ciento. 


\section{CARACTERISTICAS FISICAS}

\begin{tabular}{|c|c|c|c|c|c|c|c|c|c|}
\hline 1 & 2 & 3 & 4 & 5 & 6 & 7 & 8 & 9 & 10 \\
\hline \multirow{2}{*}{$\begin{array}{c}\text { SONDEO } \\
\text { № }\end{array}$} & \multirow{2}{*}{ 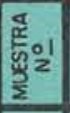 } & \multirow{2}{*}{ COTA $\mathrm{m}$. } & \multirow{2}{*}{$\Phi$} & \multirow{2}{*}{$\begin{array}{l}\text { HUMEDAD } \\
\text { NATURAL }\end{array}$} & \multicolumn{3}{|c|}{ LIMITES ATERBERG } & \multirow{2}{*}{$\begin{array}{c}\text { DENSIDAD } \\
\text { APARENTE SECA }\end{array}$} & \multirow{2}{*}{$\begin{array}{l}\text { COMPRE- } \\
\text { SION } \\
\text { SIMPLE. } \\
\text { KG./cm? }\end{array}$} \\
\hline & & & & & L.L. & L.P. & I.P. & & \\
\hline 1 & 1 & $3.60-4.00$ & 5.4 & 15.3 & 44 & 16 & 28 & 1.69 & 2.8 \\
\hline 1 & 2 & $9.50-10.00$ & 3.5 & 23.7 & 48 & 20 & 28 & 1.52 & 5 \\
\hline 2 & 1 & $1.85-2.25$ & 5.4 & - & - & - & 一 & - & - \\
\hline 3 & 1 & $1.60-2.00$ & 5.4 & 16.2 & 51 & 19 & 32 & 1.80 & 3.3 \\
\hline 3 & 2 & $4.90-5.30$ & 5.4 & 23.5 & 55 & 22 & 33 & 1.55 & 2.5 \\
\hline 3 & 3 & $9.75-10.20$ & 3.5 & 23.4 & 59 & 21 & 38 & 1.56 & 5.9 \\
\hline 3 & 4 & $14.50-1500$ & 3.5 & 25.6 & 49.5 & 20.5 & 29 & 1.51 & 4.3 \\
\hline 5 & 1 & $4.45-4.85$ & 5.4 & - & - & - & - & - & - \\
\hline 10 & 1 & $2.05-2.55$ & 54 & 22.7 & 59 & 20 & 39 & 1.60 & 3.8 \\
\hline 10 & 2 & $3.72-4.22$ & 5.4 & 10.6 & 38 & 18 & 20 & 1.91 & 2.5 \\
\hline 10 & 3 & $11.73-12.23$ & 3.5 & 22.2 & 53 & 21 & 32 & 1.60 & 27 \\
\hline 10 & 4 & $21.00-21.50$ & 3.5 & 21.2 & - & - & - & 1.70 & 8.6 \\
\hline 10 & 5. & $24.60-25.10$ & 3.5 & 20.6 & - & - & - & 1.71 & 10.3 \\
\hline II & 1 & $2.00-2.50$ & 5.4 & - & 53 & 20 & 33 & - & - \\
\hline II & 3 & $10.00-10.50$ & 5.4 & - & 44 & 21 & 23 & - & - \\
\hline "I & 4 & $15.00-15.50$ & 5.4 & - & 40 & 20 & 20 & - & - \\
\hline 12 & 1 & $2.00-2.50$ & 5.4 & - & 42 & 18 & 24 & - & - \\
\hline 12 & 3 & $9.50-10.00$ & 5.4 & - & 45 & 21 & 24 & - & - \\
\hline 18 & 1 & $6.50-7.00$ & 3,5 & 22.1 & 55 & 25 & 30 & 1.72 & 7.7 \\
\hline 18 & 2 & $12.00-12.50$ & 3.5 & 21.3 & 49 & 19.5 & 295 & 1.77 & 9.5 \\
\hline 22 & 1 & $5.00-5.50$ & 3.5 & 25.6 & 54 & 22 & 32 & 1.69 & 4.8 \\
\hline 22 & 2 & $15.00-15.50$ & 3.5 & 20.5 & - & - & - & 1.81 & 9 \\
\hline- & - & - & - & $\rightarrow$ & - & $\ldots$ & - & - & - \\
\hline
\end{tabular}

\section{Resistencia a compresión} simple

La resistencia a compresión simple se ha realizado con muestras inalteradas de $30 \mathrm{~mm}$ de diámetro. Los resultados son crecientes con la profundidad, y la forma de la rotura de las probetas es plástica en la mayoría de los casos, obteniéndose valores más bien elevados (columna 10, cuadro I).

\section{Compresibilidad \\ e hinchamiento}

A fin de estudiar y medir estos efectos en los tres tipos de arcillas que forman el subsuelo (amarilla, gris y azul), se tomaron sendas muestras inalteradas en su estado natural.

El ensayo de compresibilidad realizado ha sido el normal en presencia de agua, lo que ha originado en el estado de humedad y densidad natural un hinchamiento de la muestra, que ha sido impedido durante el ensayo con incrementos de cargas sucesivas; por cuya razón las curvas edométricas representadas en el sistema semilogarítmico tienen la primera parte de la rama descendente paralela al eje de la presión, hasta el estado de carga que equilibra la presión de hinchamiento con la sobrecarga. En la figura 11 se pueden observar las curvas representativas del fenómeno correspondientes a las muestras más características.

Por tanto, estos suelos tienen propiedades expansivas muy acusadas, así como de contracción, siendo, en consecuencia, muy sensibles a las variaciones de humedad. Por esta causa habremos de identificarlos cuantitativamente.

Es notorio comprobar que los daños que se observan en los edificios son muy diferentes, aun cuando sus dimensiones sean idénticas, posean análoga profundidad de cimentación y los suelos ofrezcan características geotécnicas similares. 
CURVA GRANULOMETRICA DE SUELOS

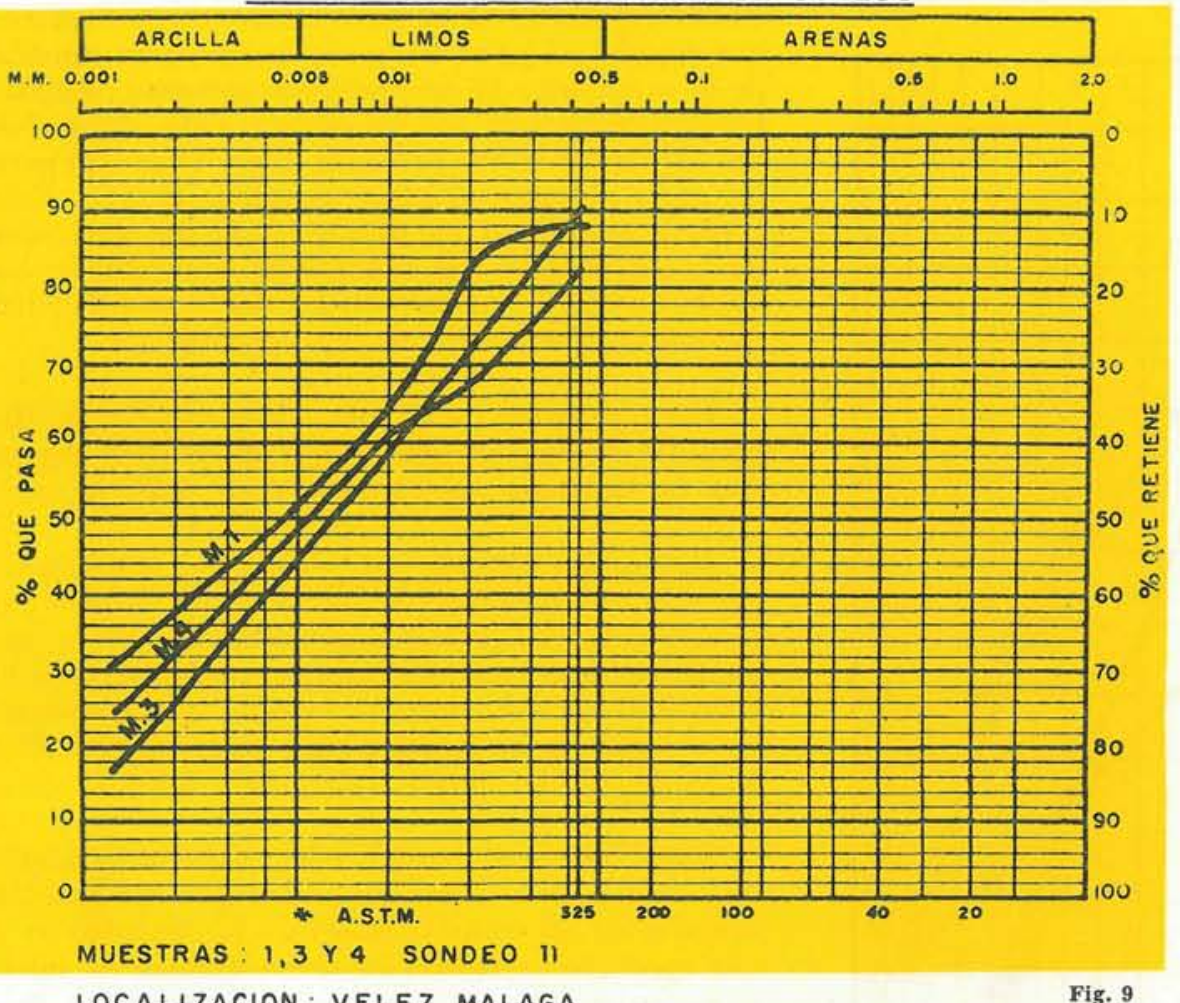

LOCALIZACION : VELEZ MALAGA
Esto es debido a que, según ha demostrado Seed en su reciente trabajo «Prediction of Swelling», dos muestras del mismo suelo, teniendo la misma densidad y \% de humedad, pero compactadas por distintos métodos, pueden ofrecer diferentes potenciales de hinchamiento. La capacidad expansiva depende, por una parte, de la estructura inicial y, por tanto, de la forma en que se actuó sobre el suelo cuando se ejecutó la cimentación, y en segundo lugar, de su grado de humedad.

Los \% de humedad que ofrecían las muestras eran elevados, como se ha indicado, por lo que nos encontrábamos próximos al límite superior de hinchamiento. En vista del comportamiento de las muestras extraídas, era conveniente buscar algunas características que, mediante los ensayos de rutina efectuados, diesen una idea del valor y de la importancia de los efectos que este fenómeno ha producido.

El valor de la actividad de las arcillas, según Skempton, viene expresado por:

$$
A=\frac{I P}{C-N} .
$$

en donde IP es el índice de plasticidad de las arcillas; $\mathrm{C}$, el $\%$ de material que pasa por el tamiz 200 de las normas ASTM, y N, constante que, en nuestro caso, adopta el valor 5. Tomando los valores correspondientes a $\mathrm{l}$ as muestras representativas, deduciremos:

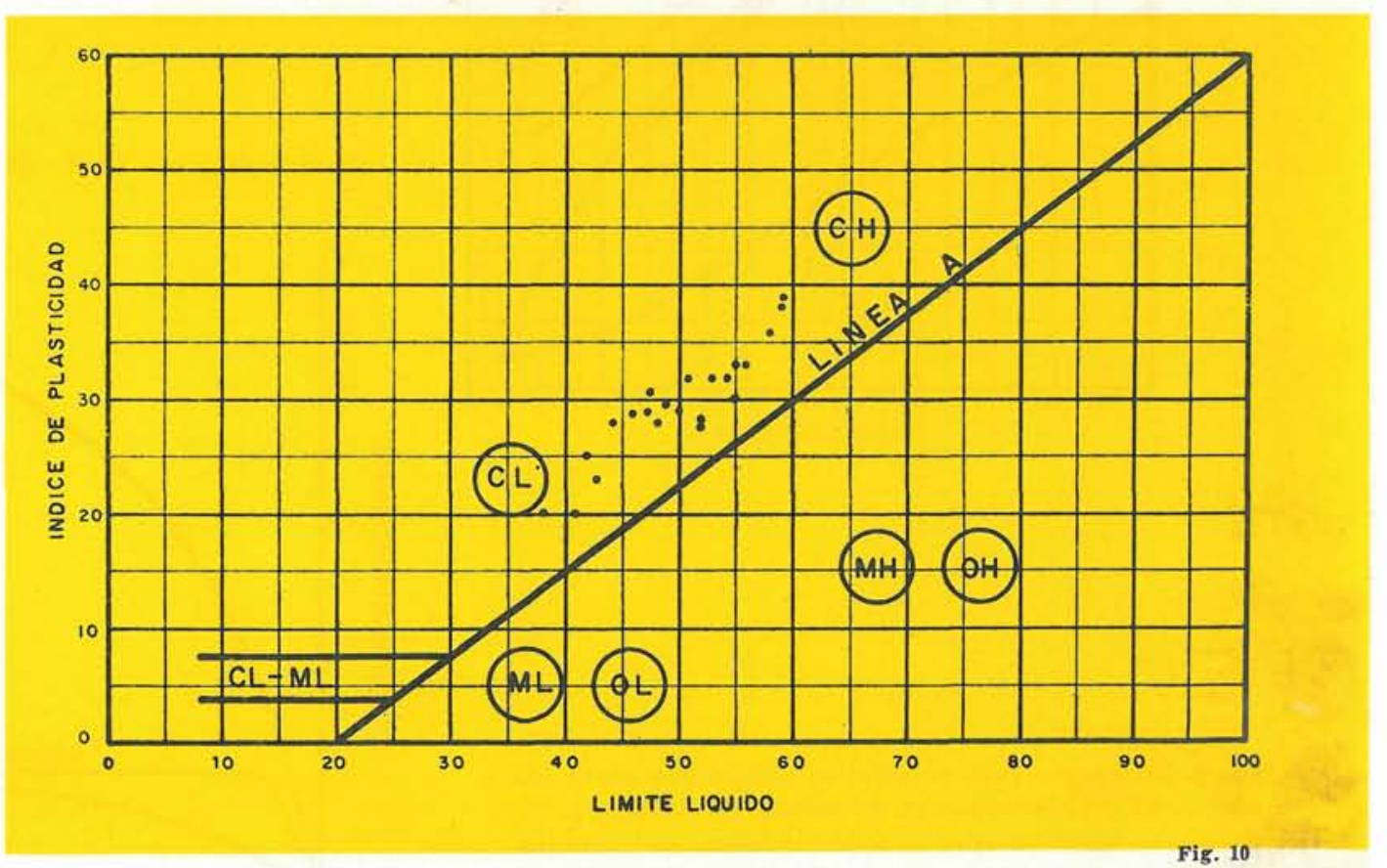

$\begin{array}{ccccc}\text { Sondeo } & \text { Muestra n. } & \text { IP } & \text { C } & \text { A } \\ 11 & 1 & 33 & 37 & 1,03 \\ 11 & 3 & 23 & 26 & 1,09 \\ 11 & 4 & 20 & 32 & 0,74\end{array}$

Para determinar el potencial de hinchamiento se sometieron las muestras al efecto de inundación, permitiéndoles la expansión libre, en sentido vertical, 


\section{currvass de consolicilacion.}

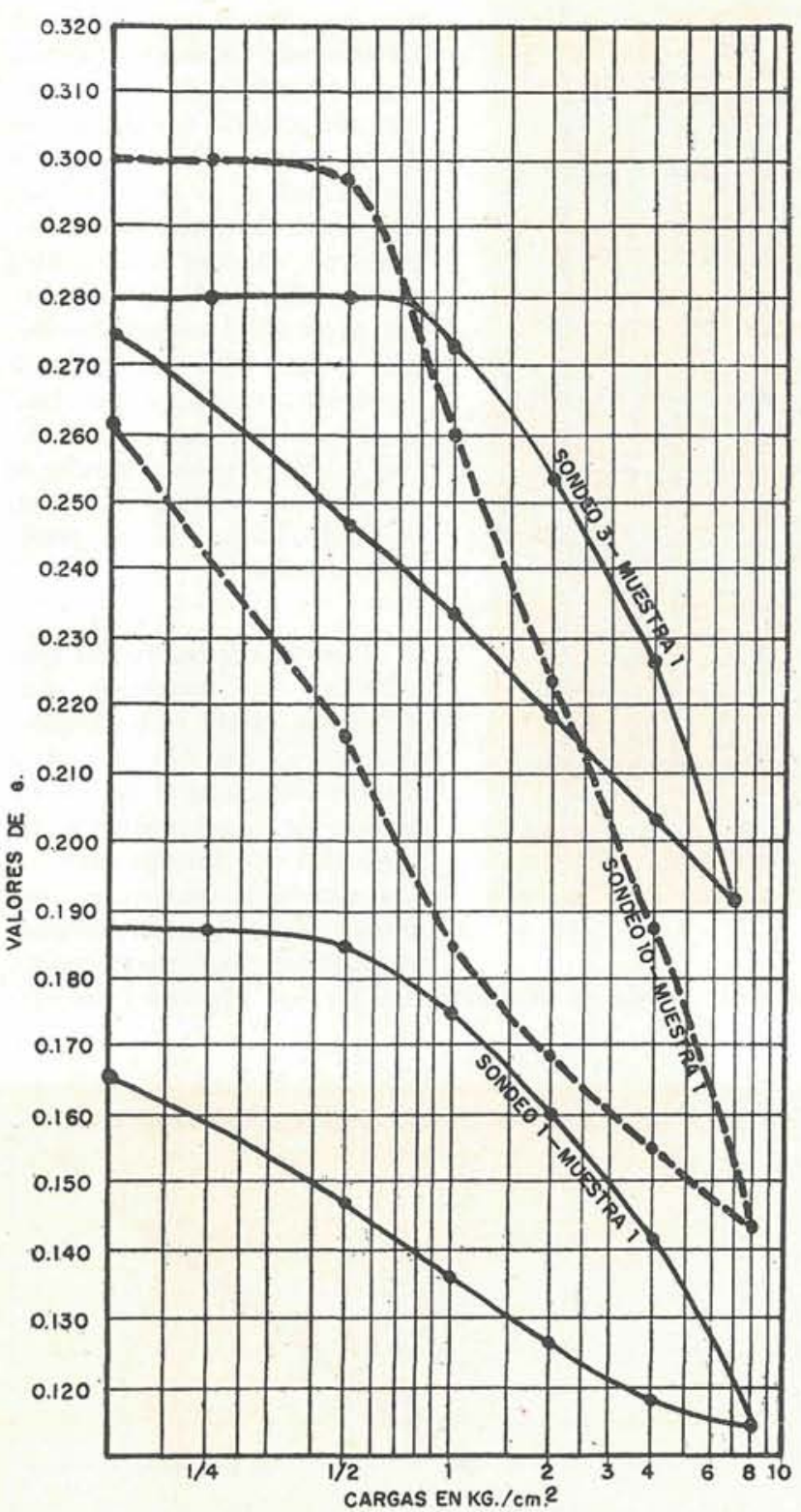

Fig. 11

sin sobrecarga. Los resultados obtenidos para las muestras representativas aparecen en la figura 12, donde se observa que el valor máximo del hinchamiento ha sido alcanzado por la muestra número 1 , la más superficial del sondeo número 11 , que supera al $9 \%$, y las otras dan valores próximos al $2 \%$, que indican se trata de expansiones alta y media, respectivamente.
De acuerdo con la clasificación que figura en el citado estudio de Seed, para los suelos particulares que estudió, el valor de la expansión como función de la actividad y \% de partículas menores de 0,002 milímetros, resulta ser, en nuestro caso, del orden del $15 \%$ para la muestra núm. 1, mientras que para las otras muestras resultan próximas al $5 \%$. Esto pone de manifiesto una desviación en los resultados de Seed, debido a la naturaleza diferente de los suelos ensayados en cada caso, y del \% de humedad natural en el momento de la extracción.

Por otra parte, de la curva de consolidación (figura 11) deducimos que la máxima presión expansiva ha quedado contrarrestada por $0,70 \mathrm{~kg} / \mathrm{cm}^{2}$,

La presión ejercida por peso propio y sobrecargas sobre el cimiento para una casa de un piso era de $0,40 \mathrm{y}$ de $0,60 \mathrm{~kg} / \mathrm{cm}^{2}$, para las de dos, por lo que quedaban unas fuerzas ascendentes máximas de 0,30 y de $0,10 \mathrm{~kg} / \mathrm{cm}^{2}$, respectivamente, sin compensar en el caso de la muestra más desfavorable. Ello ha producido los graves daños observados.

Estas presiones perjudiciales, todavía se incrementaban con la acción expansiva que por fricción actuaba en las caras laterales de la cimentación y que agravaban aún más las circunstancias. Al sufrir el suelo las sucesivas acciones expansivas, su módulo de elasticidad disminuye con las consiguientes consecuencias que se derivan del caso.

\section{ensage de hinchammiemto}

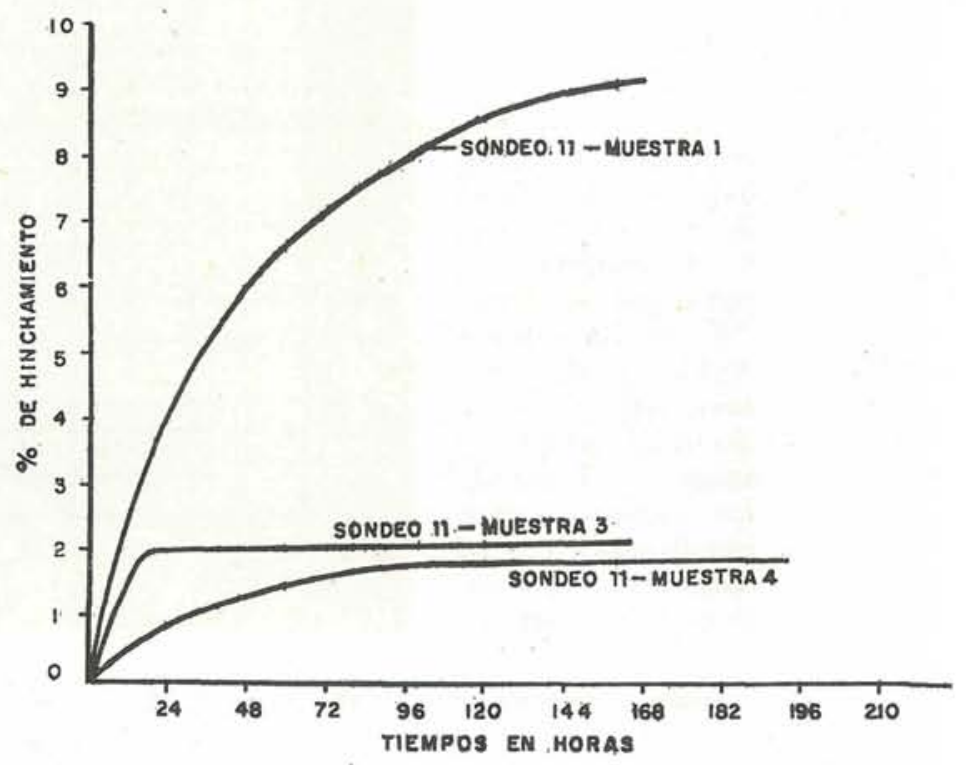

Fig. 12 


\section{EDmelusiomes}

En esta zona costera de la provincia de Málaga son muy frecuentes los casos de existencia de suelos expansivos, y se han escogido los situados al SE. de Vélez-Málaga para realizar sobre ellos un estudio detallado en el presente trabajo. Sobre dichos suelos están edificados unos bloques de viviendas que se encuentran gravemente dañados, como consecuencia de la acción de los mismos sobre su cimentación, daños que se ofrecen a la vista del observador.

La constitución arcillosa de tales suelos es debida a la formación geológica del pizarral silúrico que la bordea. El suelo consiste en unas arcillas azules que adoptan la coloración grisácea en su primera fase de alteración para terminar amarillentas. La oxidación avanza en profundidad.

De los análisis roentgenográficos efectuados se deduce que estas arcillas están constituidas, fundamentalmente, por ilita, carbonato cálcico y cuarzo en la proporción de $1: 2: 3$, además de montmorillonita, dolomita y otros elementos. Así, pues, la ilita es el mineral arcilloso predominante, hecho que ha sido confirmado por el análisis térmico diferencial y, por tanto, es el elemento que ha provocado los efectos expansivos del suelo. Naturalmente que la acción de la ilita ha quedado reforzada por la presencia de la montmorillonita y la dolomita.

La potencia de hinchamiento se observa más elevada en los estratos superiores, correspondientes a las arcillas amarillas, que en las azules de base. Este hecho es, en parte, debido a la riqueza de carbonato cálcico que posee la arcilla azul, y que llega a ser superior al doble de la superficial. La abundancia de $\mathrm{CO}_{3} \mathrm{Ca}$ en la arcilla azul reduce su índice de plasticidad $\mathrm{y}$, por tanto, disminuye la actividad expansiva del suelo.

El valor máximo deducido para el potencial de hinchamiento en el Laboratorio, ha sido del $9 \%$ en la muestra número 1, superficial del sondeo núm. 11, y en el resto, valores sensibles. Estos valores originaron en la cimentación de las edificaciones correspondientes, importantes esfuerzos verticales. Las presiones ejercidas por el cimiento eran del orden de 0,40 a $0,60 \mathrm{~kg} / \mathrm{cm}^{2}$, que, naturalmente, no quedaban compensadas con la acción expansiva, la cual, en el caso de la muestra más desfavorable, alcanzaba valores de $0,70 \mathrm{~kg} / \mathrm{cm}^{2}$. Este fenómeno ha sido el productor de los daños observados.

Se han fijado las siguientes fases que constituyen la solución de cimentación de las nuevas edificaciones que se proyecta construir en estos suelos:

1) Excavar hasta penetrar $1 \mathrm{~m}$ en la marga azul, de características menos activas.

2) Poner en contacto con la marga, gravas que habrán de constituir una zona de neutralización de las futuras acciones expansivas.

3) Este estrato de grava tendrá un espesor suficiente para absorber la expansión volumétrica calculada del suelo.

4) Cimentar sobre la grava apisonada, con hormigón armado.

5) Verter arcilla no activa apisonándola de forma que envuelva lateralmente a la fábrica de cimentación, con objeto de impedir la entrada de agua en su base, que pudiera poner a la marga azul en actividad y, por otra parte, que absorba y neutralice los esfuerzos tangenciales de posibles acciones verticales originadas por el suelo.

Estas indicaciones habrán de servir de guía para el recalce de las construcciones existentes. 


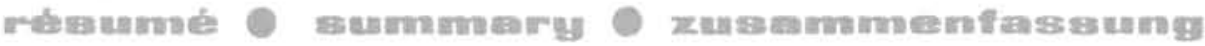

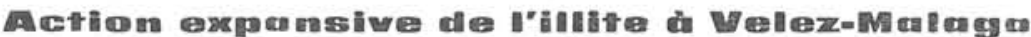

F. Oliveros, Ing. des Ponts et Chaussées.

La zone côtière centrale de la province de Malaga offre fréquemment des cas d'existence de sols expansifs. Ceux situés au sud-est de qui souffrent de graves dommages, visibles pour n'importe quel observateur, à cause de l'action de ces sols sur les fondations.

La constitution argileuse de ces sols est due à la formation géologique de l'ardoisière silurienne qui l'environne. Le sol se compose d'argiles bleues qui adoptent une coloration grisâtre au cours de leur première phase d'altération pour devenir flnalement jaunâtres. rofondeur.

Des analyses roentgénographiques effectuées on déduit que ces argiles sont constítuées fondamentalement d'illite, de carbonate caleique argileux prédominant, fait qui a été confirmé par l'analyse thermique différentielle. C'est donc là l'élément quí a provoqué les effets expansifs du sol.

On observe une puissance de gonfiement plus élevée dans les strates supéricures, correspondantes aux argiles jaunes que dans les strates correspondantes aux argiles bleues de base. Ce fait est dû, en partie, à la richesse de carbonate calcique que possède l'argile bleue, qui arrive à être supérieure au double de l'argile superficielle. L'abondance de CO.Ca dans l'argile bleue réduit son indice de plasticité La valeur maximum déduite pour le potentiel de gonflement en laboratoire a été du $9 \%$ et, pour le reste, des valeurs appréciables. Ces valeurs ont provoqué d'importants efforts verticaux dans les fondations des édifices correspondants. Les pressions exercées par ces fondations étaient de Tordre de 0,40 a $0,60 \mathrm{~kg} / \mathrm{cm}^{2}$, qui, naturellement, n'etaient pas compensées avec 1 action expansive qui, dans le ca

Comme solution de fondations, il est done recommandé de les exécuter en béton armé sur la marne bleue, appuyé sur une couche de pierres consolidée qui absorberaient l'expansion volumétrique du sol, neutralisant ainsi ces effets, s'ils venaient à se produire. Les fondations doivent être protégées par une argile non active qui exercerait une action imperméable, évitant ainsi l'entrée des eaux dans la base, ce qui pourrait provoquer le début de leur processus de gonflement.

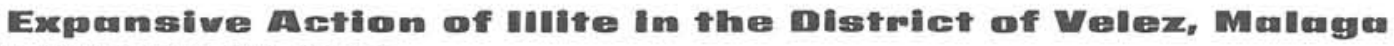 \\ F. Oliveros Rives, civil engineer.}

Along the central coastal region of Malaga there are frequent cases of expansive soils, and those to the south east of Vélez-Malaga, have been chosen to make a detailed study of them in this article. Some blocks of dwellings have been built on these soils. These blocks are severely damaged, as a result of the action of these soils on the foundations. This damage can be easily observed.

The clay composition of these soils is due to the geological formation of the Siluric slates that surround them. The soil is made up of blue clays, which are grey in their initial phase of development, and finally become yellow. Oxydation progressively penetrates more deeply.

$X$ ray analysis shows that these clays are mainly made up of illite, calcium carbonate and quartz, in the ratio of $1: 2: 3:$. They also contain montmorilonite, dolomite and other products. Thus the illite is the main clay mineral. This fact has been confirmed by diffe-

The expansive process appears to be greater in the top layers, that correspond to the yellow clays, than in the bottom, blue, layers. This fact is partly due to the rich content of calcium carbonate in the blue clay, which can be as much as twice the content at the surface. The abundance of $\mathrm{CO}_{3} \mathrm{Ca}$ in the blue clay reduces its plasticity index, and consequently decreases the expansive action of the soil. The maximum value obtained at the laboratory for the expansion coefficient has been $9 \%$ : over the rest of the zone noticeable values have been recorded. These magnitudes meant that the foundations of the buildings in question were subjected to important vertical forces. The pressure exerted by the foundation varied between 0.4 and $0.6 \mathrm{~kg} / \mathrm{cm}^{2}$. Which naturally, was not compensated with the expan-
sive pressure. This, in the most unfavourable case reached the value of $0.75 \mathrm{~kg} / \mathrm{cm}^{2}$. This phenomenon has been the eause of the observed damages.

As a solution it has been proposed to build reinforced concrete foundations on the blue clay, and place them on compacted gravel. The latter would absorb the volumetric expansion of the soil, thus neutralizing their action, if it should take place. The foundation should be protected by non active clay, which should serve as a waterproof protection against the access of water to the base, since this might
initiate an expansive process.

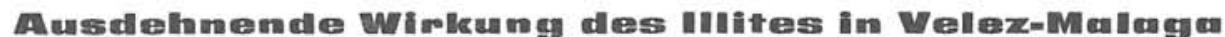

F. Oliveros Rives, Bauingenieur.

In der mittleren Küstenzone der Provinz Malaga sind die Vorkommen von Schwellungsböden sehr häufig. Man hat die im So von Velez-Malaga ausgewählt, um in der vorliegenden Arbeit ein eingehendes Studium über sie anzustellen. Auf den genannten Böden wurden einige Wohnblöcke erbaut, welche schwer beschädigt sind, der Wirkung der genannten Böden auf die Fundierung zufolge. Derart sind die Schäden, dass sie der Beobachter sehen kann.

Die tonige Beschaffenheit solcher Böden wird durch die geologische Formation des silurischen Schiefers bedingt, der sie umgibt. Der Boden besteht aus blauen Schiefern, die im ersten Stadium der Umbildung die gräuliche Färbung annehmen, um dann gelblich zu werden. Die Oxydation schreitet nach der Tiefe fort.

Aus den rhoentgenographischen Analysen leitet man ab, dass diese Tone grundsätzlich aus Illit, Kalziumkarbonat und Quarz, im Verhältnis $1: 2: 3$, gebildet werden, ausserdem aus Montmorillonit, Dolomit und anderen Elementen. Es ist also der Illit das vorherrschende tonige Mineral, eine Tatsache, die durch die therm
ausdehnenden Kräfte des Bodens hervorgerufen hat.

Die Schwellungskraft beobachtet man in grösserem Masse in den oberen Schichten, die den gelben Tonen entsprechen, als an den lauen im Grunde. Diese Tatsache ist teilweise durch den Reichtum an Kalzium-Karbonat, das der blaue Ton enthält, bedingt, der ber doppelt so gross als an der Oberfiäche ist. Der Ueberfuss an $\mathrm{CO}_{3} \mathrm{Ca}$ im blauen Ton reduziert seine Anzeichen von Plastizität und

Der abgeleitete Höchstwert für die Schwellungskraft im Laboratorium betrug $9 \%$ und im übrigen sinnfälige Werte. Diese Werte verursachten bei der Fundierung der entsprechenden Gebäude bedeutende senkrechte Kräfte. Der von der Fundierung ausgeübte Druck schwankte von 0,40 bis $0,60 \mathrm{~kg} / \mathrm{cm}^{2}$, was natürlich nicht die ausdehnende Wirkung ausglich, welche im ungünstigsten Falle Werte von $0,75 \mathrm{~kg} / \mathrm{cm}^{2}$ erreichte. Diese Erscheinung hat die beobachteten Schäden erzeugt.

Als Lösung für die Fundierung empfiehlt man, mit Stahlbeton auf dem blauen Mergel zu gründen und auf gestampftem Kies, der die Volumenausdehnung des Bodens absorbiert und so derartige Wirkungen, falls sie auftreten, neutralisiert. Die Fundierung muss durch inaktiven Ton geschützt werden, der eine undurchlässige Wirkung ausübt und den Eintritt des Wassers in den Grund verhindert. Dies könnte nämlich den Beginn seines Schwellungsprozesses hervorrufen. 